\title{
Associations between changes in sleep and body composition in African American children
}

\begin{abstract}
Objective: Eating, exercising but also sleeping occurs within the socio-cultural niche in which the child develops, likely exerting a major influence on their well-being. Sleep of underserved children is not well-documented despite their acknowledged health risk. We hypothesized that poor sleep will be adversely associated with body composition.
\end{abstract}

Study design: A cross-sectional sequence designed study centered on a 14day objective sleep recording with concomitantly assessed anthropometric measures.

Setting: Children aged 3 to 9years old living in the south side of Chicago area were invited to participate in the study. Findings reflect a sample of 24 children.

Instruments and outcome measures: Each child wore an actiwatch for 24hours per day during three separate two-week periods spread over three months. Changes in sleep were associated to changes in body composition.

Main findings: Increased sleep duration and increased number of sleep bouts, associated with weight loss, improved linear growth, slimmer waist and hip circumferences or a more proportional body composition, especially after six weeks. Restless naps, and specifically napping variability, as well as variable wake after sleep onset of naps, adversely related to hip circumference at four weeks, and waist circumference and its ratios at six and 12 weeks, respectively. Results further showed that the time to fall asleep, and the time to fully awaken, played a peculiar role in the somatic growth of the child.

Conclusions: More and regular quality sleep resulted in a healthier somatic growth pattern.

Implications: Leptogenic home-interventions should include sleep onset and offset, fragmentation of sleep in addition to sleep duration.

Keywords: sleep, nap, African American, child, body mass index, actigraphy
Volume 2 Issue 3 - 2018

\author{
Karen Spruyt,' Calista U Alaribe, ${ }^{2}$ Odochi U \\ Nwabara $^{2}$ \\ 'Lyon Neuroscience Research Center, University Claude \\ Bernard, France \\ ${ }^{2}$ Department of Health Studies, Chicago State University, USA
}

Correspondence: Karen Spruyt, Lyon Neuroscience Research Center, INSERM U I028-CNRS UMR 5292-Waking Team, University Claude Bernard, School of Medicine, Lyon, France, Email karen.spruyt@inserm.fr, karen.spruyt@lyon I.fr

Received: March 03, 2018 | Published: July II, 2018
Abbrevations: SPT, sleep period time; TST, total sleep time; SEI, sleep efficiency index; SONL, sleep onset latency; WASO, wake after sleep onset; SOFL, sleep offset latency; SLBOUTS, sleep bouts, FRAGIN, fragmentation index

\section{What is known about the topic}

a. Sleep assessment is progressively more incorporated in health research.

b. Mounting evidence suggests that sleep is a modifiable risk factor in relation to childhood obesity.

c. Body mass index has been the most prominent player in studies examining the relationship between sleeps and weight problems.

\section{What this paper adds}

a. This study is the first to measure sleep (daytime and nighttime) objectively and longitudinally in an underserved sample of young children.

b. Findings highlight that healthcare professionals should look beyond sleep duration and body mass index across childhood.

c. Health care professionals, and society, should promote the integrity of sleep in childhood.

\section{Introduction}

Studies have indicated that minority children are at an increased health risk. An odds ratio of 4.64 for obesity in African American children living on the south side of Chicago was reported. ${ }^{1}$ After controlling for factors known to be associated with increased prevalence of obesity, only their race or ethnicity and household income remained significant.

Obesity research in general has been emphasizing energy intake and expenditure in terms of cause and cure, with a concomitant shift in interventions focusing on the home-environment. Besides eating, exercising also sleeping occurs within the socio-cultural niche in which the child develops. Sleep plays an important role in health, and its role in the regulation of metabolism and obesity risks is welldebated. ${ }^{2-5}$ Alternatively, per the 2010 National Sleep Foundation poll, African American adults, in comparison to other ethnicities, have fewer hours of sleep and have sleep habits and attitudes that diminish the likelihood of optimal sleep. ${ }^{6}$ Recently it was reported that $72 \%$ of African Americans experience sleep problems. ${ }^{7}$ Given that a child's sleep is dependent upon parental attitudes and habits, ${ }^{8,9}$ sleep of a minority child might be at significant risk.

Findings suggested that short sleep combined with a variable sleep pattern across 7-days is associated with an adverse metabolic profile in school-aged children. ${ }^{10}$ In adolescents high sleep variability was 
found to be related to higher energy intake and snack consumption. ${ }^{11}$ The causal mechanisms between poor sleep and weight problems are thought to be complex. That is, on the one hand, prior sleep is known to relate to changes in the sleep architecture of subsequent sleep. ${ }^{12,13}$ On the other hand, also napping is known to affect nighttime sleep and its structure. ${ }^{12-14}$ The pathways between poor sleep and weight problems might therefore be multi-layered. For instance, after sleep deprivation slow-wave activity in non-rapid eye movement sleep is increased, decreasing immediately after a recovery night; whereas an early evening nap, rich in slow-wave sleep, diminishes the subsequent nighttime slow-wave activity. ${ }^{12,13}$ Noteworthy, and as an example, during slow wave sleep the human growth hormone is released. ${ }^{15}$ Although it remains uncertain whether sleep deprivation or altered sleep architecture affects metabolic homeostasis, the potential paralleling of both poor sleep with a dramatic increase in prevalence of obesity at any age is extensively debated. ${ }^{16-19}$

Sleep, and its (co)morbidities, in a sample of predominantly minority children are not well documented, albeit the acknowledged health risk in underserved populations. ${ }^{20}$ That is, primarily post-hoc analyses of survey data showed that African American children tend to nap more, longer and till an older age..$^{21-23}$ Lately pediatric sleep research is progressively exploring ethnic differences ${ }^{24-26}$ and the need for repeated objective sleep monitoring in the home is clear. We reported that in young African American children, across 14days, sleep is a daily challenge, with sleep occurring as a need and a chance within an ephemeral 24hour society. ${ }^{27}$

Given the lack of sleep information in underprivileged children, and the high odds of obesity in children living on the south side of Chicago, repeated measurement over a prolonged period might aid better understanding of their health risk. We therefore hypothesized that poor sleep of African American children, living in the south side Chicago area, will adversely relate to their body composition.

\section{Methods}

\section{Participants}

This study was approved by the Institutional Review Board of The University of Chicago (IRB 10-677-B) and participating community centers on the South Side of Chicago, with obtained informed consent from parents, and when applicable assent from children. Boys and girls were 3 to 9years of age, lived in the south side of Chicago area and were self-defined as being African Americans.

\section{Instruments}

Sleep: The Actiwatch-2 (Philips-Respironics. Inc. 2009, version 5 ) is a small non-dominant wrist accelerometer for sleep monitoring. Sleep intervals over a 24 hour period were manually marked based on parental logs and clinical experience. ${ }^{28,29}$ Sleep parameters of interest were: Sleep Period Time (SPT), Total Sleep Time (TST), Sleep Efficiency Index (SEI) which is (TST/SPT)*100, Sleep Onset Latency (SONL), Wake After Sleep Onset (WASO), Sleep Offset Latency (SOFL), Sleep Bouts (SLBOUTS), Fragmentation Index (FRAGIN). All sleep parameters (except the SONL, SOFL, FRAGIN and SLBOUTS) were calculated through an enhanced software algorithm, applying polysomnography-derived correction factors and using statistics from surrounding major rest periods, to improve the sleep statistics for a particular sleep interval recorded by actigraphy(http:// actigraphy.respironics.com/resources.aspx).
Sleep data is expressed in terms of mean and variability (i.e., how variable the child sleeps relative to its individual mean), and this for: 1) nighttime sleep, daytime sleep or napping, and overall sleep (i.e., nighttime and daytime combined $)^{27}$; 2) weekdays, weekend days (Friday-Saturday), and the 14-days or the full recording period; and 3) week-weekend difference.

\section{Body composition}

Measurements were repeated three times, and measurement was done at the start/the end of each recording period. Average values per recording period will be utilized in the statistical analyses. Height (HEIGHT, meter) was measured to the nearest $1 \mathrm{~mm}$ with a portable stadiometer (Charder HM200P Portstad Portable Stadiometer) and body weight (WEIGHT, $\mathrm{kg}$ ) was measured with the participant dressed in light clothing to the nearest $0.1 \mathrm{~kg}$ using a weighing scale (Life source UC-321 Precision Personal Health Scale). Body Mass Index (BMI, and BMI z-score), Height-for-age z-score (HAZ), Weight-forheight (WHZ) and Weight-for-age z-score (WAZ) were calculated via CDC Epi Info (Epi Info ${ }^{\mathrm{TM}}$ Version 3.5.3). Waist circumference (WAIST) was measured standing, at the level of the uppermost lateral border of the iliac crest, and hip circumference (HIP) at the level of the greater trochanter with a non-stretchable fiberglass tape (MyoTape). Based on the measurements we generated Waist-to-Hip Ratio (WHIR), Waist-to-Height Ratio (WHER), Body Surface Area (BSA) by the Mosteller equation, and Body Fat Percentage (BF\%) $\left[(1.51 * \mathrm{BMI})-\left(0.70^{*} \mathrm{Age}\right)-(3.6 *\right.$ gender $)+1.4$, where male gender $=1$, female $=0] \cdot{ }^{30-32} \mathrm{~A}$ BMIZ $\geq 1.65$ was considered as fulfilling the criteria for obesity, while a BMIZ $>1.04$ was defined as overweight and a BMIZ $<-1.04$ as underweight. Given that there is no unified way to express body composition of developing children, and alternatives to the BMI are being developed, we determined to include several 'surrogate' parameters.

\section{Procedure}

Through a cross-sectional sequence designed study, centered on a 14day objective sleep recording with concomitantly assessed anthropometric measures, we recruited children from the south side of Chicago. Namely, the child wore an actiwatch for 24 hours per day during three separate two-week periods (T1, T2 \& T3) spread over three months (Figure 1). Simple random sampling within the participating community centers was applied. Inclusion criteria were African American child between the ages of 3 to 9years old with a primary caregiver fluent in English language and having telephone access. The Kish procedure ${ }^{33}$ for subject selection was used when several children within the family were eligible for the study. Participants were excluded when parents reported that children had sleep problems (e.g., sleep apnea), medical illness (e.g., diabetes), developmental disabilities, psychiatric disorders or were taking medications on a regular basis. Furthermore, the Behavioral Risk Factor Surveillance System Questionnaire ${ }^{34}$ was partially implemented to screen and exclude the primary caregiver in terms of medical illness, psychological disorders, health problems and drug use. Per recording period, participants received a \$25 gift card (Figure $1)$.

\section{Data analysis}

Prior to the inferential analyses discussed in the current study, General Linear Modeling was performed on the repeated sleep and body measures (not reported); their Observed Power ranged from 0.51 
(variability of the FRAGIN) to 0.99 (SPT), which is commonsense given the device and sleep parameter (with alpha set at 0.05$).{ }^{27}$

Individual change scores were calculated. The change score is a statistical technique for measuring change that includes the examination of the level of initial performance, and addresses the statistical phenomenon of regression to the mean in terms of repeated measurements. It is therefore a preferred method for assessing change over time; i.e., a positive change indicates your score increases and a negative change indicates your score decreases with time. At the same time, change scores are clinically meaningful and allow direct comparisons; i.e., scores beyond \pm 1.96 (95\% Confidence Interval (CI)) are considered clinically significant (or a true change). In layterms, a change score expresses (true) change within the individual over time.

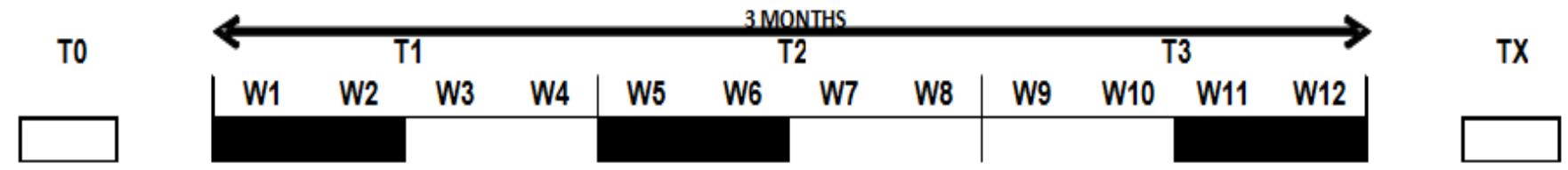

Figure I Timeline of the sleep recordings.

T: time;T0, screening moment;TI-3 are months enrolled in the study; TX, feedback moment; W: week

Interested families were contacted to set a date for the first month (TI) to start recording, which was individually determined. The black box represents the sleep recording.

Pearson (r) and Spearman ( $\left.r_{s}\right)$ correlation analyses express the associations between sleep and body composition change scores. Correlations with $\mathrm{p}$-values $<0.01$ will be considered as significant and will be reported. To ascertain a power of 0.8 , at this p-level in a sample of 24 , correlations can go as low as 0.5 . In addition, a curve analysis with distance-weighted least square fitting procedure was applied, revealing non-salient overall patterns of data. Statistical analyses were performed with statistica version 10 (Stat Soft, Inc. (2009), STATISTICA, Tulsa, OK).

\section{Results}

Children ( $\mathrm{n}=24)$ were $5.4 \pm 1.7$ (95\%CI: 4.6-6.1) years old of which 13 were girls $(54.2 \%)$. Children's average Total Sleep Time was insufficient (per National Sleep Foundation recommendations) over three months; i.e., 6.51 hours at night and at daytime 1.42 hours, and was qualitatively variable. ${ }^{27}$ The socio demographic features of the current sample reflect those of participants to a previous large-scale survey; for this reason we believe that the current sample might be considered to be representative of that population.

\section{Body composition}

Over three months children increased in HEIGHT and WEIGHT. Overall WAIST, HIP and subsequent ratios WHIR, WHER remained invariant. Average $\mathrm{BF} \%$ was similar, whereas BSA significantly increased. The generated $z$-scores were similar over the three recording periods: HAZ, WAZ, WHZ and BMI or BMIZ. As a result, $50 \%$ of the children were of normal weight; i.e., $4.2 \%$ was underweight, $25 \%$ was overweight and $20.8 \%$ was obese (Table 1 ).

Table I Body Composition of the sample at the first sleep monitoring period ( $\mathrm{TI}$ ), the second sleep monitoring period (T2), and the third sleepmonitoring period (T3)

\begin{tabular}{|c|c|c|c|c|c|}
\hline & $\begin{array}{l}\text { TI } \\
\text { mean } \pm \text { SD } \\
95 \% \mathrm{Cl}\end{array}$ & $\begin{array}{l}\text { T2 } \\
\text { mean } \pm \text { SD } \\
95 \% \mathrm{Cl}\end{array}$ & $\begin{array}{l}\text { T3 } \\
\text { mean } \pm S D \\
95 \% \mathrm{Cl}\end{array}$ & Test-statistic & p-value \\
\hline Height & $\begin{array}{l}1.176 \pm 0.13 \\
1.12-1.23\end{array}$ & $\begin{array}{l}\text { I. } 179 \pm 0.14 \\
\text { I.12-1.24 }\end{array}$ & $\begin{array}{l}1.189 \pm 0.13 \\
1.13-1.25\end{array}$ & $F(2,46)=29.5$ & $<0.0001$ \\
\hline Weight & $\begin{array}{l}26.2 \pm 11.7 \\
21.28-31.13\end{array}$ & $\begin{array}{l}26.5 \pm 11.8 \\
21.49-31.42\end{array}$ & $\begin{array}{l}26.9 \pm 11.8 \\
21.88-31.88\end{array}$ & $F(2,46)=33.9$ & $<0.0001$ \\
\hline Waist & $\begin{array}{l}0.59 \pm 0.1 \\
0.55-0.63\end{array}$ & & & $F(2,46)=0.04$ & 0.96 \\
\hline Hip & $\begin{array}{l}0.68 \pm 0.12 \\
0.63-0.73\end{array}$ & & & $F(2,46)=0.89$ & 0.42 \\
\hline Waist-to-Hip ratio & $\begin{array}{l}0.9 \pm 0.1 \\
0.85-0.89\end{array}$ & & & $F(2,46)=0.007$ & 0.99 \\
\hline Waist-to-Height ratio & $\begin{array}{l}0.5 \pm 0.1 \\
0.48-0.52\end{array}$ & & & $F(2,46)=0.90$ & 0.42 \\
\hline Body Fat percentage & $\begin{array}{l}23.3 \pm 5.8 \\
20.9-25.8\end{array}$ & & & $F(2,46)=1.09$ & 0.35 \\
\hline Body Surface Area & $\begin{array}{l}0.915 \pm 0.24 \\
0.81-1.02\end{array}$ & $\begin{array}{l}0.921 \pm 0.24 \\
0.82-1.02\end{array}$ & $\begin{array}{l}0.932 \pm 0.24 \\
0.83-1.03\end{array}$ & $F(2,46)=55.9$ & $<0.0001$ \\
\hline
\end{tabular}




\begin{tabular}{|c|c|c|c|c|c|}
\hline & $\begin{array}{l}\text { TI } \\
\text { mean } \pm \text { SD } \\
95 \% \mathrm{Cl}\end{array}$ & $\begin{array}{l}\text { T2 } \\
\text { mean } \pm S D \\
95 \% \mathrm{Cl}\end{array}$ & $\begin{array}{l}\text { T3 } \\
\text { mean } \pm S D \\
95 \% \mathrm{Cl}\end{array}$ & Test-statistic & p-value \\
\hline Height for Age Z-score & $\begin{array}{l}0.81 \pm 1.38 \\
0.23-1.4\end{array}$ & & & $F(2,46)=2.8$ & 0.07 \\
\hline Weight for Age Z-score & $\begin{array}{l}1.05 \pm 1.2 \\
0.5-1.55\end{array}$ & & & $F(2,46)=2.26$ & 0.12 \\
\hline Weight for Height Z-score & $\begin{array}{l}0.59 \pm 0.82 \\
0.16-1.02\end{array}$ & & & $F(2,30)=0.18$ & 0.83 \\
\hline Body Mass Index & $\begin{array}{l}18.13 \pm 3.82 \\
16.52-19.74\end{array}$ & & & $F(2,46)=0.09$ & 0.91 \\
\hline Body Mass Index Z-score & $\begin{array}{l}0.95 \pm 1 \\
0.53-1.38\end{array}$ & & & $F(2,46)=0.52$ & 0.60 \\
\hline
\end{tabular}

Over the three months, individual changes were measured, yet Figure 2 shows that no clinical significant change in body composition was seen for the total sample.

\section{Associations between changes in sleep and body composition}

Differential associations between sleep and body composition were found across each of the recording periods (Scheme 1) (Table
2). All correlations are $\geq 0.5$ and results are reported in a synthesized manner across the scheme.

SOFL showed the highest number of correlations, especially regarding the nighttime and the overall sleep period. Generally, longer SOFL associated with increased WEIGHT, WAIST, HIP, WHIR, WHER, BSA, and BF\%. These associations were primarily found on the short (after 4weeks) and long term (after 12weeks). Particularly, the mean SOFL and its differences in Week-Weekend days stand out.

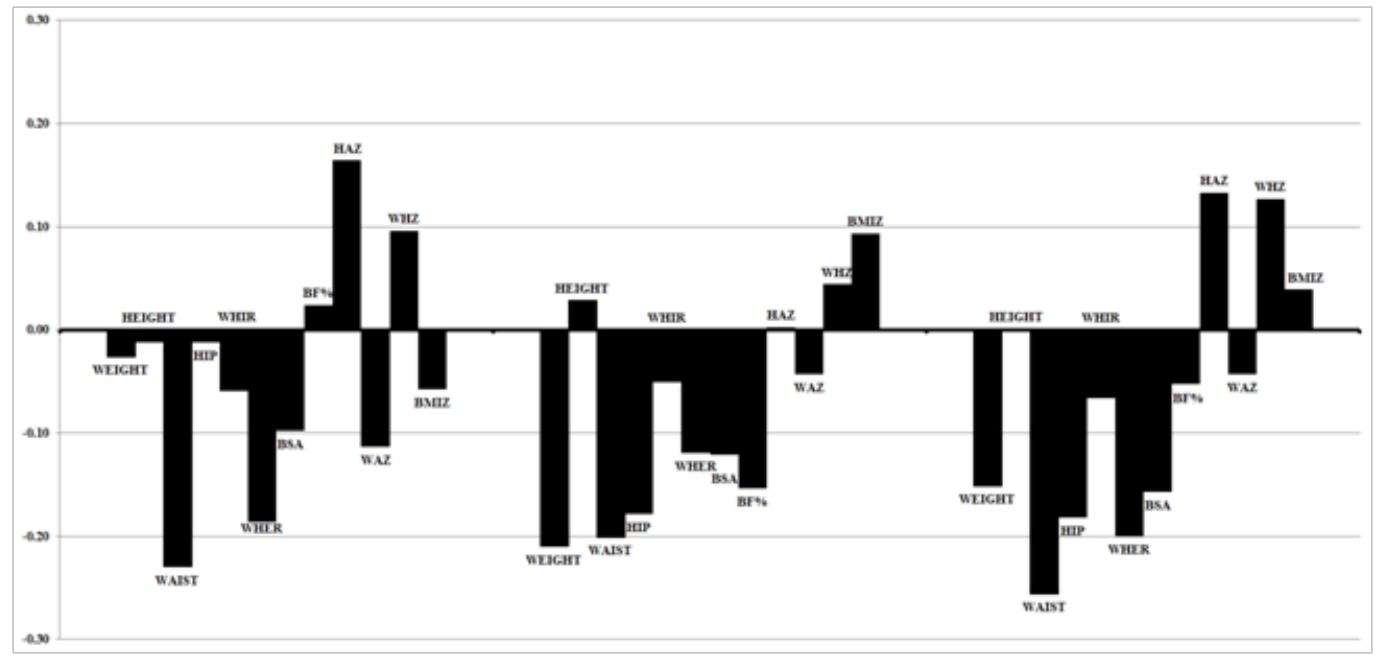

Figure 2 Change scores of Body Composition over three months (each $n=24)$.

Left: change after four weeks Middle: change after six weeks Right: change after 12 weeks of interest, in the sample positive changes (i.e., more than initially) were seen for BF\%, HAZ,WHZ after four weeks, for HEIGHT, HAZ,WHZ, BMIZ after six weeks, and finally for HAZ,WHZ and BMIZ after I2weeks. WAIST, waist circumference, HIP, hip circumference, WHIR, Waist-to-Hip ratio, WHER, Waist-to-Height ratio, BSA, Body Surface Area, BF\%, Percentage Body Surface Area, HAZ/WAZ/WHZ, Height for Age z-score, Weight for Age z-score, Weight-to-Height Z-score, BMIZ, Body Mass Index Z-score.

Next, in young African American children, especially hip circumference and waist circumference were found to be susceptible to changes in sleep. Namely, regarding HIP, on the short (after 4weeks) and the middle long term (after 6weeks) associations between HIP and sleep were found, and they were mostly positive correlations. For the nighttime, and also the overall sleep period, especially WASO (or the sleep quality) related to HIP. For the daytime period, this association was mostly with its TST (or the sleep duration). Overall average sleep 'integrity' as expressed by the mean of the sleep parameters such as WASO, SLBOUTS, TST, SEI stand out. Regarding WAIST, the majority of associations were found on the middle long (after 6weeks) and long term (after 12weeks). Again, primarily positive correlations were found with sleep, and more specifically with SOFL. Although mostly average sleep (or the means of the parameters) associated, also variability in sleep was found to be correlated with WAIST.

Most of the negative correlations were found between SLBOUTS (or the number of epochs scored as sleep) and body composition. These associations were predominantly found for sleep during the daytime period, and on the long term (after 12weeks).

In terms of sleep variability and its association with body composition of young African children, we found that variably TST on the middle long term (after 6weeks) and variably FRAGIN on the long term (after 12weeks), along with primarily WAIST associated. 
Table 2 Associations between changes in sleep and body composition

\begin{tabular}{|c|c|c|c|c|c|c|}
\hline \multirow[t]{2}{*}{ \# in scheme } & \multicolumn{2}{|c|}{ After 4 weeks } & \multicolumn{2}{|c|}{ After 6 weeks } & \multicolumn{2}{|c|}{ After 12 weeks } \\
\hline & correlation & p-value & correlation & p-value & correlation & p-value \\
\hline I & 0.65 & 0.005 & -0.57 & 0.004 & 0.52 & 0.009 \\
\hline 2 & 0.56 & 0.005 & -0.61 & 0.002 & 0.60 & 0.002 \\
\hline 3 & -0.52 & 0.009 & 0.65 & 0.001 & -0.55 & 0.005 \\
\hline 4 & 0.61 & 0.002 & 0.55 & 0.006 & -0.52 & 0.009 \\
\hline 5 & 0.64 & 0.006 & 0.57 & 0.003 & -0.55 & 0.005 \\
\hline 6 & -0.56 & 0.005 & -0.54 & 0.007 & $-0.59 *$ & 0.008 \\
\hline 7 & -0.66 & 0.003 & 0.60 & 0.002 & $-0.66^{*}$ & 0.002 \\
\hline 8 & -0.65 & 0.004 & -0.53 & 0.007 & $-0.69 *$ & 0.001 \\
\hline 9 & 0.63 & 0.007 & -0.69 & $<0.0001$ & $0.64 *$ & 0.006 \\
\hline 10 & $0.93 *$ & $<0.0001$ & 0.62 & 0.001 & $0.65^{*}$ & 0.004 \\
\hline II & $-0.89 *$ & 0.006 & 0.60 & 0.002 & $-0.96 *$ & 0.0004 \\
\hline 12 & $-0.93^{*}$ & 0.003 & 0.53 & 0.008 & -0.74 & 0.004 \\
\hline 13 & 0.53 & 0.008 & 0.54 & 0.006 & 0.58 & 0.003 \\
\hline 14 & -0.55 & 0.005 & 0.56 & 0.004 & 0.56 & 0.005 \\
\hline 15 & 0.53 & 0.008 & $0.7 I^{*}$ & 0.001 & 0.61 & 0.002 \\
\hline 16 & 0.55 & 0.005 & $0.65 *$ & 0.005 & 0.61 & 0.001 \\
\hline 17 & $-0.96 *$ & $<0.0001$ & $0.7 \mid *$ & 0.001 & 0.55 & 0.005 \\
\hline 18 & & & $0.8 I^{*}$ & 0.0001 & 0.63 & 0.001 \\
\hline 19 & & & $-0.76^{*}$ & 0.004 & 0.58 & 0.003 \\
\hline 20 & & & $0.97^{*}$ & 0.005 & 0.62 & 0.001 \\
\hline 21 & & & 0.60 & 0.002 & 0.65 & 0.001 \\
\hline 22 & & & 0.64 & 0.001 & 0.56 & 0.004 \\
\hline 23 & & & -0.52 & 0.009 & 0.54 & 0.006 \\
\hline 24 & & & 0.56 & 0.004 & -0.65 & 0.001 \\
\hline 25 & & & 0.54 & 0.007 & $-0.89 *$ & 0.002 \\
\hline 26 & & & 0.62 & 0.001 & $-0.94 *$ & 0.005 \\
\hline
\end{tabular}

From TI to T2: 4weeks; from T2 to T3: 6 weeks; from TI to T3: I 2weeks; *: Spearman correlation (unmarked are Pearson correlations).

Body composition in general associated mostly positively with nighttime sleep, on the middle (after 6weeks) and long term (after 12 weeks). In contrast, mostly negative correlations were found between body composition and daytime sleep, on the middle (after 6 weeks) and long term (after 12weeks).

Lastly, average nighttime sleep was found to be associated with body composition particularly on the longer term, whereas variably daytime sleep associated with body composition on the short, middle and long term.

Graphically the longitudinal interrelation between somatic growth and sleep is presented in Figure 3. Both panels of Figure 3 represent the non-linear association between changes in 14-days overall sleep and the common used BMIZ. Each of these may suggest the importance of the synergy between regular and sufficient sleep, as well as the timing of sleep in childhood.

\section{Discussion}

Findings predominantly indicated that changes in sleep over time were associated with changes in somatic growth, yet not in a unitary or linear fashion. Results further underlined that sleep onset and especially offset combined with the quality of sleep were influential. Also, the timing of sleep within the 24-hour rhythm showed a differential impact on body composition. More and regular quality sleep resulted in a healthier somatic growth pattern. Future studies may investigate the synergy between sleep duration, regularity and timing towards treating the obesity epidemic in childhood.

To the best of our knowledge, this is the first longitudinal study in underserved children that objectively recorded 14day 24hour sleep, with a concomitant anthropometric assessment and an extra focus on napping. Previous studies have shown that children in our society are likely to obtain insufficient sleep. ${ }^{35-38}$ African American children are further suggested to exhibit more gradual age-related 
decline in napping; i.e., they napped significantly more, had shorter average nocturnal sleep durations, and slept significantly less on weekdays than on weekend nights when compared to Caucasian at age 2 to 8years. ${ }^{39}$ Poor sleep was found in young African American children. ${ }^{27,40,4}$ Children in the Chicago area additionally have higher obesity rates than low-income children of a similar age in the U.S. ${ }^{42}$

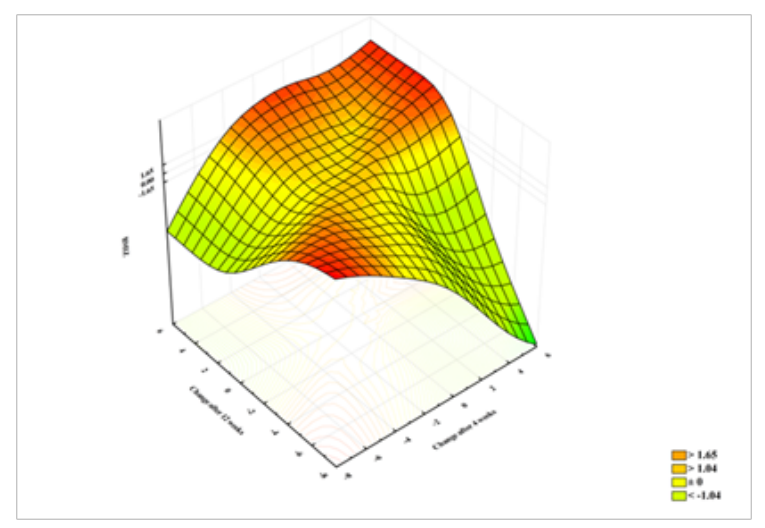

Figure 3 Distance-weighted least square fitting of the change scores of total sleep time and final Body Mass Index z-score $(n=24)$

Panel IA Left: 14-Days overall sleep: change from A to B (4weeks) and from $A$ to $C$ (I 2 weeks) Sleeping continuously less or more was associated (Xand Y-axis) with higher BMI Z-score (Z-axis). Invariant sleep time [i.e., without clinical significant change in sleep after 4 or 12 weeks or within \pm 2 area of $X$-and Y-axis being the center of the plot] was associated with overweight BMI z-score (orange). Sleeping more (X-axis) yet not continuously (Y-axis) was associated with lower BMI z-scores.

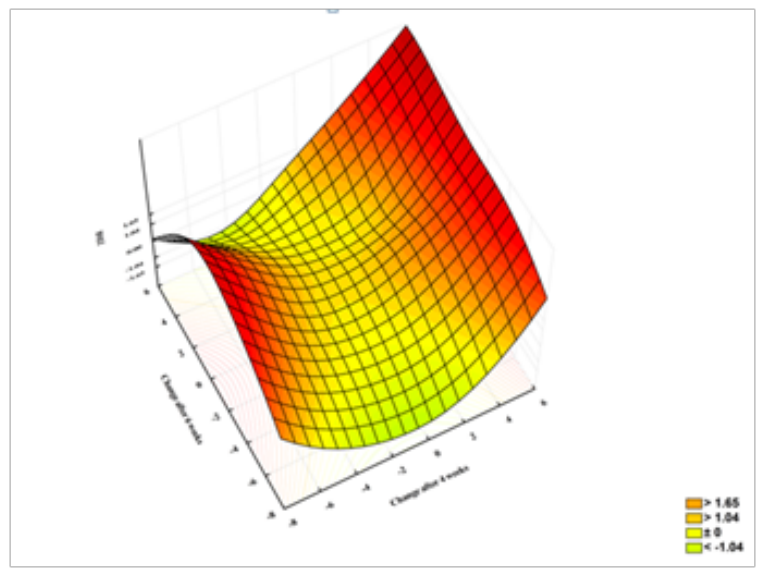

Panel IB Right: I4-Days overall sleep: change from A to B (4weeks) and from B to C (6weeks)

Sleeping more after 4weeks (X-axis), irrespective of the amount of sleep during the following period (yet continuously more is higher risk, Y-axis), was associated with higher BMI z-score. This is similar when sleeping less after 4weeks (X-axis), especially when kept consistent (Y-axis zero point) higher BMI z-score is associated (orange/red).

In this study, more and regular sleep across any time-point either expressed as increased sleep duration or increased number of sleep bouts, improved body composition. Namely, weight loss, improved linear growth, slimmer waist and hip circumferences-or a more proportional body composition-, especially after six weeks, was found. In this sample, insufficient sleep, or stated differently as a tendency for weekend "catch-up" as well as napping "catch-up", was previously inferred..$^{27}$ The health impact of this 'sleep-coping' behavior became especially apparent after 12 weeks, where changes in the week-weekend sleep duration adversely affected somatic growth. In other words, it is not merely the time spent in bed but the time spent to good quality sleep at regular times at nighttime over a prolonged period which might be protective. More specifically, on the one hand, age-appropriate napping was found to be beneficial towards a healthy somatic growth. But, on the other hand, findings after 12 weeks suggested that a consistent nighttime sleep from Monday to Monday might be preferred. Our findings are in line with the possible rebound of sleep, or the changes in sleep architecture of subsequent sleep by poor sleep of the previous period (i.e., increase of a sleep parameter above baseline levels after a period of poor sleep). ${ }^{12,13}$ That is, fragmented or restless naps, and especially napping variability, as well as variable wakening after sleep onset during napping, were found to adversely impact the hip circumference at four weeks, and waist circumference and its ratios at six and 12 weeks respectively. Indeed, children were found to consistently sleep less than recommended, with little variability in nighttime sleep duration apart from napping opportunities. ${ }^{27}$ As a result, "catch-up" sleep behavior could be a double-edged sword.

Actually, improving sleep efficiency which is a rough indicator of the time spent to sleep while in 'bed' might be a crude 'rule of thumb'. That is, regarding napping already within four weeks, improved sleep efficiency decreased body fat percentage. Whereas over a longer period with respect to nighttime sleep, benefits towards the hip circumference and linear growth were found. Perhaps more slow-wave sleep, or better proportioned overall sleep architecture, might be assumed. These are intriguing findings in light of continuing knowledge about the rebound of sleep in general.

Results also showed that the time to fall asleep or sleep onset latency, and the time to fully awaken or sleep offset latency, play a peculiar role in the somatic growth of the child. Namely, both 'short' and 'long' may be adverse; for instance, a short sleep onset latency may be indicative of sleep deprivation (e.g., being extremely tired) versus a long sleep onset latency may be suggestive of a lacking opportunity to fall asleep undisturbed (e.g., noise, light, or factors in inner city neighborhoods etc.). Weekly short sleep onset latency at four weeks increased the body fat percentage, whereas at 12 weeks it also increased waist circumference and waist-to-height ratio. Short sleep offset latency might indicate sufficient sleep or interrupted sleep period, vice versa for a long sleep offset latency. Within the sleepobesity debate, sleep onset and offset are consistently ignored despite their clinical relevance.

Notwithstanding the lack of polysomnographic data, our findings are supportive of the role of the sleep-wake cycle, and its homeostasis, with respect to the somatotropic axis. ${ }^{43,44}$ Although more objective studies are needed, one pediatric study found a negative association between rapid eye movement sleep and obesity. ${ }^{45}$ Children are known to spend proportionally more time in slow-wave sleep at the beginning of their sleep period, and more time in rapid eye movement sleep at the end of their sleep period. Even so, and in light of the rebound of sleep, the role of sleep onset and offset needs to be further investigated, given its predominance after six and 12 weeks when changes (i.e., possibly catch-ups) in the week-weekend discrepancy were analyzed. Specific to our chronic sleep deprived sample, ${ }^{46}$ it is suggested that sleep is insufficient and possible constrained by "sociocultural niche" factors, ${ }^{26,41}$ hypothetically increasing their health risk. Namely, in light of the 'sleep onset/offset latency' findings or 
alternatively the 'longer sleep during the weekend' findings, neither might be of the "best quality" sleep. Indeed, when this week-weekend discrepancy extended, somatic growth was adversely impacted. Healthcare professionals should therefore be mindful of sleep-coping behaviors.

\section{Limitations}

The actiwatch, a digitally-integrated measurement device of gross motor activity was used to visualize the rest/activity patterns, or sleep/wake, and therefore one of the limitations is the absence of sleep architecture. Despite overnight polysomnography being the widely accepted objective measure of sleep, the actigraph has some advantages; it is relatively inexpensive, minimally disruptive, can be worn continuously in natural environments. In combination with a sleep log, e.g., marking bedtime, wake-up time etc., recordings can be scored. Studies involving comparisons with polysomnographic data show adequate validity and reliability of the device. ${ }^{47}$ Next, external generalizability of findings remain limited to a young African American sample, which was in socio-demographic terms comparable to a larger underserved minority sample. ${ }^{48}$ More importantly, the sample was without risk factors given our inclusion criteria. Sleep is discussed in detail elsewhere ${ }^{46}$ and half of the sample was of normal weight. As a result, a typical developing child is being portrayed. Potential confounders, such as diet and environmental factors impacting sleep (e.g. noise), in the interrelation between sleep-body compositions are discussed elsewhere. Finally, this study is limited to anthropometric measures such that the underlying metabolic mechanisms remain speculative.

\section{Conclusion}

To summarize, our findings concur with the insufficient-excessive sleep debate or curvilinear association between sleep and weight problems. Our findings, suggestive of disparate somatotropic vectors and chronic dysregulation of sleep, may generate innovative sleepimpact pathways which should be further explored to address the endemic obesity in childhood. Health care professionals should therefore focus on the integrity of sleep over a prolonged period.

\section{Human subjects approval statement}

All procedures were in accordance with the ethical standards of the institutional and/or national research committee and with the 1964 Helsinki declaration and its later amendments or comparable ethical standards.

Informed consent was obtained from all individual participants included in the study.

\section{Acknowledgements}

Each of the participating families, the participating community centers and their representatives, Philips-Respironics, and especially my students Calista and Odochi for their enduring and successful efforts throughout this project.

\section{Conflict of interest}

The authors have no conflict of interest.

\section{Contributions}

K. Spruyt was Principal Investigator of the study: designed, supervised, analyzed and wrote the manuscript. Ms. C. Alaribe and O. Nwabara were students recruiting, maintaining and assessing participants in the study. This work was performed when the first author worked at the Department of Pediatrics, The University of Chicago, Chicago, Illinois, USA.

\section{References}

1. Margellos-Anast H, Shah AM, Whitman S. Prevalence of obesity among children in six Chicago communities: findings from a health survey. Public Health Rep. 2008;123(2):117-125.

2. Spruyt K, Gozal D. The underlying interactome of childhood obesity: The potential role of sleep. Child Obes. 2012;8(1):38-42.

3. Van Cauter E, Holmback U, Knutson K, et al. Impact of sleep and sleep loss on neuroendocrine and metabolic function. Horm Res. 2007;67(Suppl 1):2-9.

4. Hakim F, Kheirandish-Gozal L, Gozal D. Obesity and Altered Sleep: A Pathway to Metabolic Derangements in Children? Semin Pediatr Neurol. 2015;22(2):77-85.

5. Miller MA, Kruisbrink M, Wallace J, et al. Sleep duration and incidence of obesity in infants, children, and adolescents: a systematic review and meta-analysis of prospective studies. Sleep. 2018;41(4).

6. http://www.sleepfoundation.org/article/sleep-america-polls/2010 sleep-and-ethnicity

7. Nam S, Whittemore R, Jung S, et al. Physical neighborhood and social environment, beliefs about sleep, sleep hygiene behaviors, and sleep quality among African Americans. Sleep health. 2018;4(3):258-264.

8. Zheng H, Berthoud H-R. Neural Systems Controlling the Drive to Eat: Mind Versus Metabolism. Physiology(Bethesda). 2008;23(2):75-83.

9. Thomson JL, Tussing-Humphreys LM, Goodman MH, et al. Infant activity and sleep behaviors in a maternal and infant home visiting project among rural, southern, African American women. Maternal health, neonatology and perinatology. 2018;4:10.

10. Spruyt K, Molfese DL, Gozal D. Sleep Duration, Sleep Regularity, Body Weight, and Metabolic Homeostasis in School-aged Children. Pediatrics. 2011;127(2):e345-352.

11. He F, Bixler EO, Berg A, et al. Habitual sleep variability, not sleep duration, is associated with caloric intake in adolescents. Sleep Med. 2015;16(7):856-861.

12. Brunner DP, Dijk DJ, Borbely AA. Repeated partial sleep deprivation progressively changes in EEG during sleep and wakefulness. Sleep. 1993;16(2):100-113.

13. Werth E, Dijk DJ, Achermann P, et al. Dynamics of the sleep EEG after an early evening nap: experimental data and simulations. Am J Physiol. 1996;271(3 Pt 2):R501-510.

14. Kurth S, Lassonde JM, Pierpoint LA, et al. Development of nap neurophysiology: preliminary insights into sleep regulation in early childhood. J Sleep Res. 2016;25(6):646-654.

15. Sassin JF, Parker DC, Mace JW, et al. Human growth hormone release: relation to slow-wave sleep and sleep-walking cycles. Science. 1969;165(3892):513-515.

16. Horne J. Short sleep is a questionable risk factor for obesity and related disorders: Statistical versus clinical significance. Bio Psychol. 2008;77(3):266-276.

17. Cappuccio FP, Taggart FM, Kandala NB, et al. Meta-analysis of short sleep duration and obesity in children and adults. Sleep. 2008;31(5):619626.

18. Chen X, Beydoun MA, Wang Y. Is sleep duration associated with childhood obesity? A systematic review and meta-analysis. Obesity (Silver Spring). 2008;16(2):265-274. 
19. Davies SK, Ang JE, Revell VL, et al. Effect of sleep deprivation on the human metabolome. Proc Natl Acad Sci USA. 2014;111(29):1076110766.

20. Johnson DA, Lisabeth L, Hickson D, et al. The Social Patterning of Sleep in African Americans: Associations of Socioeconomic Position and Neighborhood Characteristics with Sleep in the Jackson Heart Study. Sleep. 2016;39(9):1749-1759.

21. Lavigne JV, Arend R, Rosenbaum D, et al. Sleep and behavior problems among preschoolers. J Dev Behav Pediatr. 1999;20(3):164-169.

22. El-Sheikh M, Sleep and Development: Familial and Socio-Cultural Considerations. Oxford University Press; United Kingdom 2011.

23. Hale L, Berger LM, LeBourgeois MK, Brooks-Gunn J. Social and demographic predictors of preschoolers' bedtime routines. J Dev Behav Pediatr. 2009;30(5):394-402.

24. Loredo JS, Soler X, Bardwell W, et al. Sleep health in U.S. Hispanic population. Sleep. 2010;33(7):962-967.

25. Wong WW, Ortiz CL, Lathan D, et al. Sleep duration of underserved minority children in a cross-sectional study. BMC Public Health. 2013;13:648.

26. Pena MM, Rifas-Shiman SL, Gillman MW, et al. Racial/Ethnic and Socio-Contextual Correlates of Chronic Sleep Curtailment in Childhood. Sleep. 2016;39(9):1653-166.

27. Spruyt K, Alaribe CU, Nwabara OU. To sleep or not to sleep: a repeated daily challenge for African American children. CNS Neurosci Ther. 2015;21(1):23-31.

28. Dayyat EA, Spruyt K, Molfese DL, Gozal D. Sleep estimates in children: parental versus actigraphic assessments. Nat Sci Sleep. 2011;3:115-123.

29. Spruyt K, Gozal D, Dayyat E, et al. Sleep assessments in healthy schoolaged children using actigraphy: concordance with polysomnography. $J$ Sleep Res. 2011;20(1 Pt 2):223-232.

30. Jackson AS, Stanforth PR, Gagnon J, et al. The effect of sex, age and race on estimating percentage body fat from body mass index: The Heritage Family Study. Int J Obes Relat Metab Disord. 2002;26(6):789-796.

31. Reilly JJ, Wilson J, Durnin JVGA. Determination of body composition from skinfold thickness: A validation study. Arc Dis Child. 1995;73(4):305-310.

32. Brook CG. Determination of body composition of children from skinfold measurements. Arch Dis Child. 1971;46(246):182-184.

33. Kish L. A Procedure for Objective Respondent Selection within the Household. Journal of the American Statistical Association. 1949;44(247):380-387.
34. Stein AD, Lederman RI, Shea S. The Behavioral Risk Factor Surveillance System questionnaire: its reliability in a statewide sample. Am J Public Health. 1993;83(12):1768-1772.

35. National Sleep Foundation. Sleep in America Poll-teens and sleep. 2006.

36. Yang CK, Kim JK, Patel SR, et al. Age-related changes in sleep/wake patterns among Korean teenagers. Pediatrics. 2005;115(Suppl 1):250 256.

37. http://www.sleepfoundation.org/article/sleep-america-polls/2004children-and-sleep

38. Matricciani LA, Olds Ts Fau, Blunden S, et al. Never enough sleep: a brief history of sleep recommendations for children. Pediatrics. 2012;129(3):1098-4275.

39. Crosby B, LeBourgeois MK, Harsh J. Racial differences in reported napping and nocturnal sleep in 2-to 8-year-old children. Pediatrics. 2005;115(Suppl 1):225-232.

40. Bates CR, Bohnert AM, Ward AK, et al. Sleep is in for summer: Patterns of Sleep and Physical Activity in Urban Minority Girls. Journal of pediatr psychol. 2016;41(6):692-700.

41. Vezina-Im LA, Hughes SO, Baranowski T, et al. Association Between Sleep Duration and Body Mass Index Among US Low-Income Preschoolers. Obesity (Silver Spring). 2017;25(10):1770-1775.

42. Consortium to Lower Obesity in Chicago Children. Prevalence of Childhood Overweight and Obesity in Chicago. 2010

43. Van Cauter E, Plat L, Copinschi G. Interrelations between sleep and the somatotropic axis. Sleep. 1998;21(6):553-566.

44. Renaville R, Hammadi M, Portetelle D. Role of the somatotropic axis in the mammalian metabolism. Domest Anim Endocrinol. 2002;23(12):351-360.

45. Liu X, Forbes EE, Ryan ND, et al. Rapid eye movement sleep in relation to overweight in children and adolescents. Arch Gen Psychiatry. 2008;65(8):924-932

46. Spruyt K, Alaribe CU, Nwabara OU. To Sleep or Not to Sleep: A Repeated Daily Challenge for African American Children. CNS Neurosci Ther. 2015;21(1):23-31.

47. Tryon WW. Issues of validity in actigraphic sleep assessment. Sleep. 2004;27(1):158-165

48. Spruyt K, Anguh I, Nwabara O. Sleep behavior of underrepresented youth. Journal of Public Health. 2014;22(2):111-120. 